\title{
Combined radiotherapy and immunotherapy-a new promising treatment approach in oncology
}

\author{
Bin S. Teh ${ }^{1}$
}

Received: 8 October 2015 / Accepted: 3 November 2015 / Published online: 19 November 2015

(C) Springer-Verlag Berlin Heidelberg 2015

This special issue of JRO focuses on an emerging promising combined treatment approach in cancer care: radiotherapy and immunotherapy. Radiotherapy is a standard of care treatment modality for cancer whereby immunotherapy has been recently shown to be effective for treating various malignancies including melanoma, non small-cell lung cancer, and others. Together, they form a promising new partnership to improve cancer cure. The immunomodulatory effects of radiotherapy have been known for many years. The abscopal effect of radiotherapy was first described in 1953: the therapeutic effect of radiotherapy was observed distant from the radiotherapy field. It was initially observed in leukemia, lymphoma, and immunologic malignancies such as renal cell carcinoma and melanoma. This effect has been recently observed more commonly since the clinical implementation of stereotactic body radiotherapy (SBRT) delivering a higher dose per fraction and a higher total biologic equivalent dose (BED). In addition, abscopal effects are now reported even more when SBRT is used in conjunction with immunotherapy such as check point inhibitors.

Massive cell death especially caused by SBRT more than conventional fractionated radiotherapy leading to increased tumor associated antigen release, upregulation of pro-inflammatory cytokines promoting $\mathrm{T}$ cell priming and activation as well as modification of the tumor microenvironment facilitating the stimulation of immune cascade, form the basis for the immunomodulatory effects of radiotherapy. Thus, radiotherapy appears to enhance the effects of immunotherapy. In fact, we are now seeing better and more durable local, regional, and systemic responses

Bin S. Teh

BTeh@houstonmethodist.org

1 Department of Radiation Oncology, Houston Methodist Hospital, Cancer Center and Research Institute, Weil Cornell Medical College, Houston, TX, USA in patients receiving both radiotherapy and immunotherapy. Articles in this issue first give an overview of abscopal effects and the combined radiation and immunotherapy approach. These are followed by site-specific reviews of this combined approach in melanoma, lung, breast, prostate, and other genitourinary malignancies. The long-term outcome of a combined immunomodulatory gene therapy (or gene-mediated cytotoxic immunotherapy) and radiotherapy clinical trial for prostate cancer reported in this issue shows safety and efficacy likely from both cytotoxic and immunologic cell kill effects from this combined treatment approach. A very important aspect of this combined radiotherapy and immunotherapy approach is the predictors of response, which was reviewed. Patient selection based on certain tumor characteristics, e.g., the PDL1 level, is important in the context of avoiding the high costs and side effects of ineffective treatment in certain patients. A low-dose total body irradiation as a cancer immunotherapy technique is also reviewed.

There are still many unanswered questions in maximizing the full potential of this novel combined radiotherapy and immunotherapy approach in oncology. These include the optimum sequencing of these two modalities, the total dose and the fraction size of radiotherapy, the type and the number of immunotherapy to combine with radiotherapy, the feasibility of combining this radio-immunotherapy with chemotherapy and or targeted therapy as well as treatment of related side effects. It is our hope that this special issue of JRO will stimulate interest among the readers and generate collaborations and implementation of various clinical trials that incorporates the combination of radiotherapy and immunotherapy-a new and potentially promising partnership in oncology.

\section{Compliance with ethical standards}

Funding None

Conflict of interest The author declares that he has no conflict of interests. 